\title{
Stationary and non-stationary fluid flow of a Bose-Einstein condensate through a penetrable barrier
}

\author{
P. Engels $*$ and C. Atherton \\ Washington State University, Department of Physics and Astronomy, Pullman, Washington 99164, USA
}

(Dated: October 31, 2018)

\begin{abstract}
We experimentally study the fluid flow induced by a broad, penetrable barrier moving through an elongated dilute gaseous Bose-Einstein condensate. The barrier is created by a laser beam swept through the condensate, and the resulting dipole potential can be either attractive or repulsive. We examine both cases and find regimes of stable and unstable fluid flow: At slow speeds of the barrier, the fluid flow is stationary due to the superfluidity of the condensate. At intermediate speeds, we observe a non-stationary regime in which the condensate gets filled with dark solitons. At faster speeds, soliton formation completely ceases and a remarkable absence of excitation in the condensate is seen again.
\end{abstract}

PACS numbers: 03.75.Kk,03.75.Lm,67.57.De,32.80.Lg

The fluid flow past an obstacle is one of the most prototypical experiments studying superfluidity. Accordingly, moving an obstacle through a superfluid has been met with considerable interest, both in the context of superfluid helium [1] as well as in the context of dilute gaseous Bose-Einstein condensates (BECs) 2]. In experiments with BECs, the role of the obstacle can be played by a laser beam that creates a dipole potential for the atoms. By moving a small, strongly repulsive dipole beam through a BEC, evidence for a critical velocity above which superfluidity breaks down was obtained [3, 4]. In those experiments, the laser was impenetrable for the atoms, and the diameter of the laser beam was chosen smaller than the size of the BEC. The atoms could therefore flow past the sides of the moving beam. The critical velocity observed in the experiments was much lower than that given by the well-known Landau criterion [5]. Vortices can be produced by such narrow obstacles, and indeed they were observed experimentally [ $[$ ] , and in numerical simulations [7]. Likewise, in the context of optical lattices, heating due to dissipative motion has been used as a tool to study superfluidity in the presence of periodic potentials [8].

In this Letter we consider a situation that is complementary to the experiments described in [3, 4]: In our case, the BEC is contained in a narrow, elongated trap and a barrier much wider than the radial extent of the BEC is moved through it (Fig. 1(a)). The atoms cannot flow past the sides of the barrier, and we use a penetrable barrier allowing atoms to flow through the barrier. The barrier is created by the dipole potential of an elliptically shaped laser beam that is swept along the long axis of the elongated BEC. Such a geometry approximates a one-dimensional problem, and it is theoretically expected that in this case solitons play a similar role to the role played by vortices in higher dimensions [9, 10, 11, 12]. Recently, shedding of solitons has also been discussed in terms of Cerenkov-radiation [13, 14, 15]. In this Letter we provide experimental evidence for the existence of several different flow regimes in the wake of the moving barrier. For slow and for fast velocities, the wake behind the moving barrier appears unperturbed. In an intermediate velocity regime solitons appear. This behavior is observed with repulsive barriers as well as with attractive potentials.

The starting point for the experiments described in this paper are elongated ${ }^{87} \mathrm{Rb}$ BECs of about 4.5 . $10^{5}$ atoms in the $\left|F=1, m_{F}=-1\right\rangle$ state, held in a Ioffe-Pritchard type trap with trapping frequencies $\left\{\omega_{x} /(2 \pi), \omega_{y z} /(2 \pi)\right\}=\{7.1,203\} \mathrm{Hz}$. The axis of weak confinement (x-axis, see Fig. 1(a)) is oriented horizontally. Evaporative cooling is performed until no thermal cloud of uncondensed atoms is visible. A laser that is far detuned from the $\mathrm{Rb}$ absorption lines is directed along the second horizontal axis (y-axis). Depending on the detuning, it can create an attractive or repulsive dipole potential for the atoms. This dipole beam can be moved along the $\mathrm{x}$-axis at various speeds, inducing flow in the BEC. The dipole beam waist in the vertical z-direction is much larger than the radial extent of the BEC so that the intensity variation of the beam along the z-direction can be neglected. The imaging direction coincides with the direction of the dipole beam. A notch filter prevents the dipole beam from being detected by the camera.

As we show in this Letter, solitons can be produced by sweeping the dipole beam through the condensate. The width of these solitons is below our spatial imaging resolution when the BEC is held in the trap. Therefore we employ a fast antitrapping technique to expand the BEC before imaging, similar to the procedure described in 16]. The expansion imaging procedure starts with a microwave adiabatic rapid passage, converting the atoms from the $\left|F=1, m_{F}=-1\right\rangle$ state to the antitrapped $\mid F=$ $\left.2, m_{F}=-2\right\rangle$ state. We then set the magnetic bias field to $48 \mathrm{G}$ and let the cloud expand for $3 \mathrm{~ms}$. During this expansion, the magnetic field is positioned such that the antitrapping potential keeps the cloud from falling under the influence of gravity. The atoms are then imaged in a 
a)

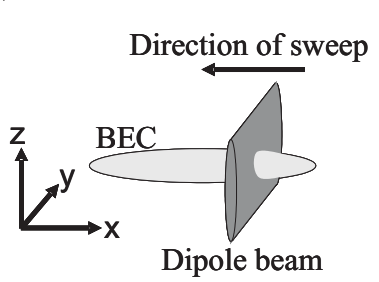

b)
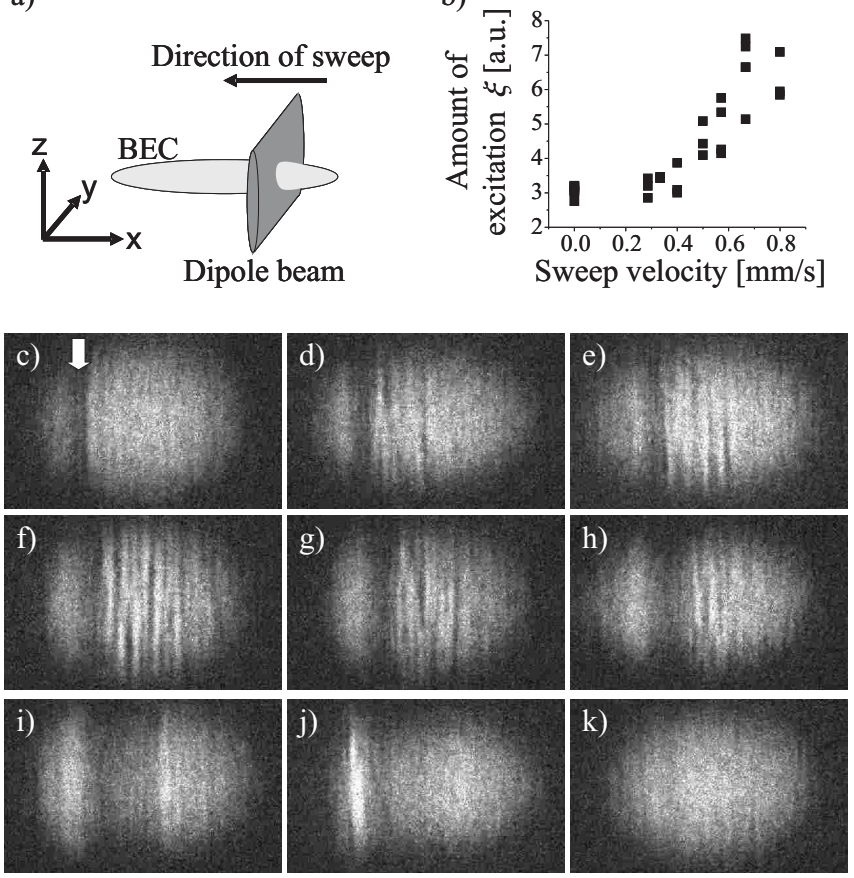

FIG. 1: (a) Axis convention. The dipole beam propagates in the $-\mathrm{y}$ direction and is moved through the BEC along the $-\mathrm{x}$ direction. (b) Onset of excitation at slow sweep speeds, calculated from images with parameters as in (c)-(k). A repulsive dipole beam is swept through the cloud from the right to the left. The white arrow in Fig. (c) indicates the end position. Sweep speeds are (c) $0.4 \mathrm{~mm} / \mathrm{s}$, (d) $0.5 \mathrm{~mm} / \mathrm{s}$, (e) $0.6 \mathrm{~mm} / \mathrm{s}$, (f) $0.7 \mathrm{~mm} / \mathrm{s}$, (g) $0.8 \mathrm{~mm} / \mathrm{s}$, (h) $1 \mathrm{~mm} / \mathrm{s}$, (i) $1.3 \mathrm{~mm} / \mathrm{s}$, (j) $2 \mathrm{~mm} / \mathrm{s}$, (k) $3.3 \mathrm{~mm} / \mathrm{s}$. Images show a $200 \mu \mathrm{m}$ wide region. Other parameters see text.

$100 \mathrm{G}$ bias field using resonant absorption imaging. The antitrapping predominantly leads to a radial expansion, so that the aspect ratio of the $\mathrm{BEC}$ changes from $R_{y z} / R_{x}$ $=29$ for the trapped BEC to a value of about 1.6 at the end of the expansion time.

In a first set of experiments, shown in Fig. 1, we study the flow induced by a weak repulsive potential swept through the BEC at various speeds. The potential was produced by a laser beam with a wavelength of $660 \mathrm{~nm}$ and a power of $37 \mu \mathrm{W}$. The beam shape was elliptical with waists $w_{x}=7.6 \mu \mathrm{m}$ and $w_{z}=25.8 \mu \mathrm{m}$. Such a laser produces a weak repulsive potential whose height is about $24 \%$ of the chemical potential of the condensate. The healing length in the center of our typical condensates is $0.17 \mu \mathrm{m}$ so that the dipole beam can be considered wide with respect to the healing length even in the direction of the sweep. The dipole beam starts out on the right, outside the BEC, and is then swept towards the left at a constant speed as given in the figure caption. The sweep is stopped at the position indicated by the white arrow in Fig.1 (c), and the BEC is imaged after antitrapped expansion as described above.

We can identify several different regimes, depending on the sweep speed. For very slow sweep speeds, such as in Fig. 1(c), the part of the BEC through which the dipole beam has been swept appears essentially unaffected, and the only visible effect of the beam is the density suppression that it leaves at its end position. Since the BEC is superfluid, it is not surprising that it remains unaffected by a slowly moving penetrable barrier. At faster sweep speeds (Fig. 1(d)-(g)), dark notches appear in the wake of the dipole beam. We interpret these notches as the solitons predicted in theoretical studies of the onedimensional problem [9, 10, 11, 12, 15] (see below for an experimental verification). Some of the notches observed in the wake of the dipole beam show slight bending or nonuniform contrast along the plane, while others are very straight and uniform. In the darkest solitons, we observe a suppression of the central density by about $50 \%$. However, it is conceivable that the true depth of some of the solitons is larger than this because slight misalignments of the imaging direction with respect to the soliton planes can reduce the apparent depth. Interestingly, fewer solitons are observed when the sweep speed is increased beyond $0.9 \mathrm{~mm} / \mathrm{s}$ (Fig. 1(h). This effect is accompanied by the appearance of an increasingly wider region of low density directly behind the dipole beam (Fig. 1(h),(i)). At faster sweep speeds, the condensate appears again unaffected by the obstacle, as seen in Fig. 1(k). The apparent absence of excitations in the wake of the beam is very striking. Suppression of phonons and solitons at high obstacle velocities was theoretically discussed in [10, 11, 17]. In particular, 11] describes an explanation of related numerical findings by using an analogy to the radiation of capillary-gravity waves in a classical fluid.

To quantify the amount of excitation in the BEC, we first calculate the smooth, overall shape of each BEC by considering only the lowest Fourier components of each image. Then the root-mean-square deviation of each original image from the smooth, overall shape is used as a measure of the excitation present in the BEC. To reduce the influence of the inhomogeneous density distribution along the $\mathrm{x}$-axis, we only consider a region in the center of the $\mathrm{BEC}$ that is $35 \mu \mathrm{m}$ wide in the $\mathrm{x}$-direction and $70 \mu \mathrm{m}$ wide along the $\mathrm{z}$-direction. The resulting quantity, $\xi$, is plotted versus the barrier speed in Fig. 1(b). The points at zero velocity are taken from unperturbed BECs in the absence of a dipole beam, and mostly indicate imaging noise. The onset of excitations above a critical velocity of roughly $0.3 \mathrm{~mm} / \mathrm{s}$ can clearly be seen in this plot. We do not extend this plot to velocities above $0.8 \mathrm{~mm} / \mathrm{s}$ because at higher speeds the broad low-density region that develops in the wake of the barrier (as seen in Fig. 1(h), (i)) affects our measure. For comparison, the bulk speed of sound in the center of our BECs is $3 \mathrm{~mm} / \mathrm{s}$. In a cigar shaped BEC, averaging over the tightly confined direction leads to a lower speed of sound [18, 19, 20]. In our case, this results in a speed of $2.1 \mathrm{~mm} / \mathrm{s}$ which 

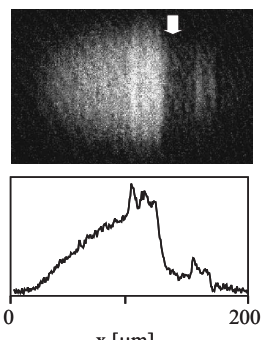

$\mathrm{x}[\mu \mathrm{m}]$
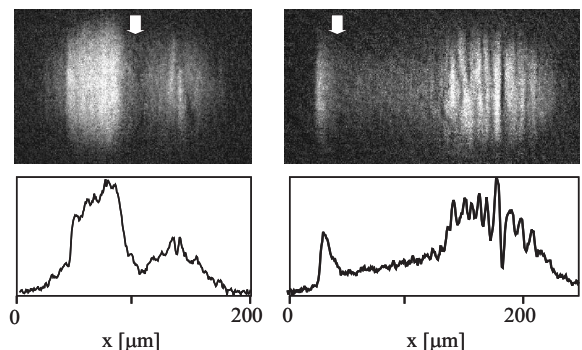

FIG. 2: Images (upper row) and corresponding integrated cross sections (lower row) for various endpoints of the sweep as indicated by the white arrows. Laserpower $69 \mu \mathrm{W}$, sweep velocity $1.4 \mathrm{~mm} / \mathrm{s}$.

is still significantly larger than the observed critical velocity. This situation is reminiscent of the observation of a surprisingly low critical velocity in vortex shedding experiments in [3, 4]. For a more precise analysis, the complicated fluid flow through the barrier would have to be taken into account. In addition, the nonuniform radial density profile of the BEC is expected to lead to a deviation from the Bogoliubov dispersion relation and to a lower critical velocity, as discussed in 21].

When a more strongly repulsive potential is used, we observe similar behaviors. However, solitons appear already at lower sweep velocities, and the velocity above which the flow becomes stationary again increases. Also, a more pronounced accumulation of atoms in front of the dipole beam is seen, delimited by a steep wavefront (Fig. 2). For weaker potentials the number of solitons in the BEC is reduced. For example, when using dipole beams with an intensity of $25 \mu \mathrm{W}$, often only one or two individual solitons are produced.

In a second set of experiments we now show that the dark notches in the wake of the dipole beam are not just soundwaves but do indeed behave like solitons. To prove this, we compare the stability of the observed notches with the stability of a simple density suppression in the BEC (Fig. 3). To obtain a very clearly visible density suppression, a relatively strong dipole beam with a power of $129 \mu \mathrm{W}$ was swept through half of the BEC. The height of the dipole potential was about $85 \%$ of the chemical potential of the condensate so that a deep density suppression was left in the BEC at the end position of the sweep. For clarity this suppression is marked by the white arrow in Fig. 3(a). The sweep velocity was adjusted to $0.2 \mathrm{~mm} / \mathrm{s}$, leading to pronounced soliton formation in the wake of the laser. At the end of the sweep, the dipole beam was switched off, and the BEC was allowed to evolve in trap for a variable evolution time before starting the expansion imaging procedure. Typical images for various evolution times are shown in Fig. 3 , At very short evolution times (Fig. 3(a)), the right part of the cloud, through which the dipole beam has been swept, shows stacks of dark notches, and the density sup-

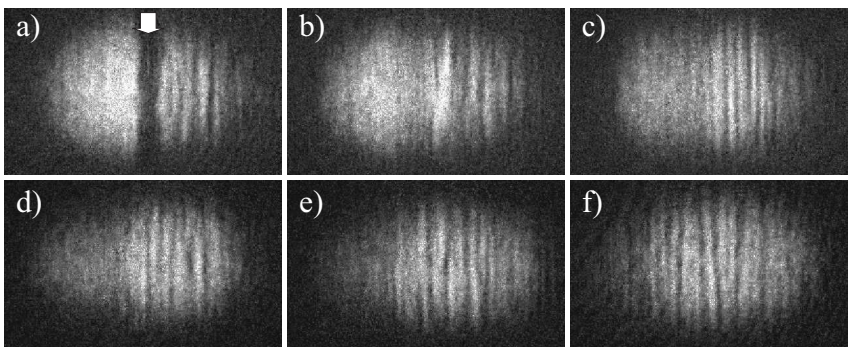

FIG. 3: Stability of the observed solitons. Times for which the BEC is allowed to evolve in trap after turning the dipole beam off at the end of the sweep and before starting the expansion imaging procedure: (a) $0 \mathrm{~ms}$, (b) $10 \mathrm{~ms}$, (c) $20 \mathrm{~ms}$, (d) $30 \mathrm{~ms}$, (e) $40 \mathrm{~ms}$, (f) $50 \mathrm{~ms}$. The arrow in (a) indicates the end position of the sweep. Other parameters see text. Images show a $200 \mu m$ wide region.

pression produced by the dipole beam at its end position is clearly visible. This density suppression gets filled in by the BEC over a time scale of about $10 \mathrm{~ms}$ (Fig. 3(b)). Considering that the longitudinal speed of sound in our cigar-shaped BEC is $2.1 \mathrm{~mm} / \mathrm{s}$, it is very plausible that the density suppression with an initial width of roughly $20 \mu \mathrm{m}$ closes during $10 \mathrm{~ms}$. However, the much narrower dark notches in the right part of the cloud remain visible for much longer times and slowly spread out (Fig. 3(b)(f)). After evolution times of over $50 \mathrm{~ms}$ they are still visible and have spread over the entire BEC (Fig. 3(f)). We often observe very straight dark notches at this time. Then their contrast gradually fades and they disappear. These measurements demonstrate the stability of the produced dark notches, suggesting that they are indeed very distinct from sound waves and correspond to the solitons predicted by theory for the one-dimensional problem.

Our condensates are elongated, but not onedimensional. Therefore, the question arises which influence the transverse dimension has on the existence of solitons. In previous experiments, individual solitons in a BEC with repulsive interactions have been engineered using phase imprinting [22, 23] or wavefunction engineering [24]. They have also been observed in the evolution of a density gap in a BEC produced by a stopped light technique 25]. One important outcome of the phase imprinting studies is that the exact nature of the solitary texture depends on the strength of the radial trapping. The numerical studies given in 26] reveal that if the chemical potential exceeds ten times the level spacing in the tightly confined direction, i.e. if $\mu / \hbar \omega_{y z}>10$, phase imprinting produces a nonstationary kink that decays into phonons on a time scale of $1 / \omega_{y z}$. If this ratio is below five, the kink decays into a proper dark soliton. Experimentally, solitons produced by phase imprinting were found in experiments with $\mu / \hbar \omega_{y z}=7$ [22]. While our mechanism for soliton formation is not based on phase imprinting, a similar influence of the transverse confinement on the existence of solitons can be expected. In particular, for 
our BECs $\mu / \hbar \omega_{y z}=9.7$, which is not too different from the experiments in 22.

By changing the laser wavelength to $830 \mathrm{~nm}$ we can also study the effects of an attractive potential, in which case the density is increased instead of decreased in the region of the potential. As in the case of repulsive potentials, theories predict soliton formation at intermediate speeds and vanishing excitation at very slow and very fast speeds [10]. We indeed observe such regimes. However, deviating from our observations with repulsive potentials, we have not found a region of suppressed density behind an attractive dipole laser that would correspond to Fig. 1(i). Results obtained with an attractive dipole beam are shown in Fig. 4. A dipole beam with a wavelength of $830 \mathrm{~nm}$ and a waist of $8.2 \mu \mathrm{m}$ in the $\mathrm{x}$-direction and $31.7 \mu \mathrm{m}$ in the z-direction was used. For the analysis in Fig. 4(a), a laser power of $10 \mu \mathrm{W}$ was chosen, leading to a potential depth of about $17 \%$ of the chemical potential of the BEC. As before we quantify the excitation by plotting $\xi$ versus the sweep speeds. Since we do not observe a low-density region corresponding to Fig. 1(i), we can now use $\xi$ as a measure for the excitation over the whole range of velocities studied. Despite the weakness of the perturbing potential, soliton formation again occurs at surprisingly low velocities.

For a laser power of $10 \mu W$, only a few solitons are produced, as shown in Fig. प(b). If the laser power is increased to $19 \mu \mathrm{W}$, the soliton formation can become quite

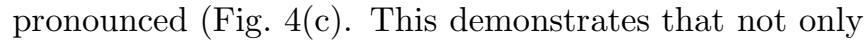
a repulsive potential, but also an attractive potential can be an efficient tool to produce solitons. One-dimensional theories for the case of attractive potentials predict the onset of soliton formation to occur right at the speed of sound [10]. However, we see that already at a speed of $1.25 \mathrm{~mm} / \mathrm{s}$, below the central speed of sound, the central part of the BEC can be filled with solitons. This discrepancy may hint at an influence of the inhomogeneous density across the condensate in our experiments.

In conclusion, we have observed stationary and nonstationary fluid flow regimes when a penetrable barrier is moved through a BEC. A slowly moving barrier leads to stationary flow. At intermediate velocities, the flow is non-stationary and is marked by the emergence of solitons in the wake of the barrier. At fast velocities, the soliton shedding stops and the flow appears stationary again. In a one-dimensional geometry, the solitons play a role analogous to vortices present in flow around obstacles in higher dimensions $[3,44,6,6]$. Thus our experiments complement these previous studies that have been hallmark experiments for investigating the superfluidity in dilute gaseous BECs.

\footnotetext{
* Electronic address: engels@wsu.edu
}

a)
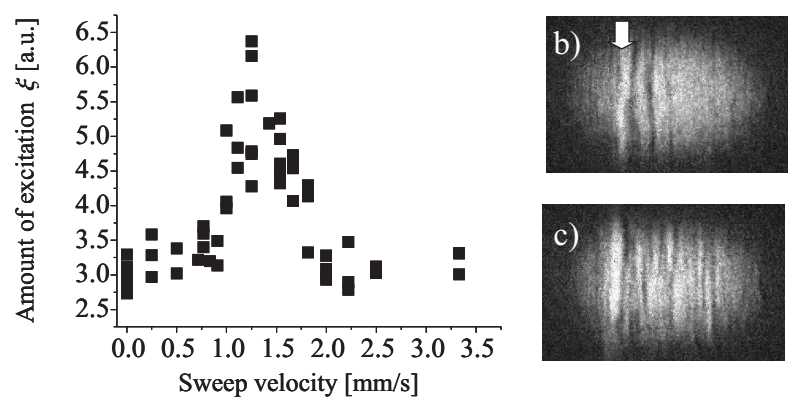

FIG. 4: Effect of an attractive potential swept through the condensate. (a) Excitation strength versus sweep speed for a laser power of $10 \mu W$, other parameters see text. (b) Laser power $10 \mu W$, sweep speed $1.25 \mathrm{~mm} / \mathrm{s}$. (c) Laser power $19 \mu W$, sweep speed $1.25 \mathrm{~mm} / \mathrm{s}$. Images show a $200 \mu \mathrm{m}$ wide region. The white arrow in (b) indicates the end position of the sweep.

[1] See, e.g., R. J. Donnelly, Quantized Vortices in Helium II (Cambridge University Press, Cambridge, 1991), and references therein.

[2] See, e.g., A. J. Leggett, Rev. Mod. Phys. 73, 307 (2001); S. Ianeselli, C. Menotti, and A. Smerzi, J. Phys. B: At. Mol. Opt. Phys. 39, S135 (2006), and references therein.

[3] C. Raman et al., Phys. Rev. Lett. 83, 2502 (1999).

[4] R. Onofrio et al., Phys. Rev. Lett. 85, 2228 (2000).

[5] L. D. Landau, J. Phys. U.S.S.R. 5, 71 (1941).

[6] S. Inouye et al., Phys. Rev. Lett. 87, 080402 (2001).

[7] T. Frisch, Y. Pomeau, and S. Rica, Phys. Rev. Lett. 69, 1644 (1992); B. Jackson, J. F. McCann, and C. S. Adams, Phys. Rev. Lett. 80, 3903 (1998);T. Winiecki, J. F. McCann, and C. S. Adams, Phys. Rev. Lett. 82, 5186 (1999), N. G. Berloff, cond-mat/0412743

[8] See, e.g., O. Morsch and M. Oberthaler, Rev. Mod. Phys. 78, 179 (2006) and references therein; B. Wu and J. Shi, cond-mat/0607098.

[9] V. Hakim, Phys. Rev. E 55, 2835 (1997).

[10] P. Leboeuf and N. Pavloff, Phys. Rev. A 64, 033602 (2001); N. Pavloff, Phys. Rev. A 66, 013610 (2002).

[11] A. Radouani, Phys. Rev. A 70, 013602 (2004).

[12] G. E. Astrakharchik and L. P. Pitaevskii, Phys. Rev. A 70, 013608 (2004).

[13] G. A. El, A. Gammal, and A. M. Kamchatnov, Phys. Rev. Lett. 97, 180405 (2006).

[14] I. Carusotto et al., Phys. Rev. Lett. 97, 260403 (2006).

[15] H. Susanto et al., cond-mat/0701435

[16] H. J. Lewandowski et al., J. Low Temp. Phys. 132, 309 (2003)

[17] C. K. Law et al., Phys. Rev. Lett. 85, 1598 (2000); M. Haddad and V. Hakim, Phys. Rev. Lett. 87, 218901 (2001); C. K. Law et al., Phys. Rev. Lett. 87, 218902 (2001).

[18] E. Zaremba, Phys. Rev. A 57, 518 (1998).

[19] S. Stringari, Phys. Rev. A 58, 2385 (1998).

[20] G. M. Kavoulakis and C. J. Pethick, Phys. Rev. A 58, 1563 (1998).

[21] P. O. Fedichev and G. V. Shlyapnikov, Phys. Rev. A 63, 045601 (2001).

[22] S. Burger et al., Phys. Rev. Lett. 83, 5198 (1999).

[23] J. Denschlag et al., Science 287, 97 (2000).

[24] B. P. Anderson et al., Phys. Rev. Lett. 86, 2926 (2001). 
[25] Z. Dutton et al., Science 293, 663 (2001).

[26] A. Muryshev et al., Phys. Rev. Lett. 89, 110401 (2002). 